\title{
CONSIDERACIONES SOBRE CALIDAD Y PRODUCTIVIDAD, UNA MIRADA DESDE LA PRAXIS ORGANIZACIONAL
}

\author{
Consequences on quality and productivity a look \\ from the organizational practice.
}

Autor de Correspondencia: Daisy Oropesa Méndez Correo:doropesa@uci.cu

\section{EPISTEMUS}

ISSN: 2007-8196 (electrónico)

ISSN: 2007-4530 (impresa)

Francisco Javier López Correa 1 Guillermo Alberto Pereira Álvarez ${ }^{2}$ Jaime Alberto Montoya Quintero 3 Diana María Montoya Quintero 4

Recibido: 12 de septiembre de 2016, Aceptado: 30 de noviembre del 2016

Autor de Correspondencia: Francisco Javier López Corre

Correo:fjlopez@elpoli.edu.co

\section{Resumen}

Se vive en una era de cambios. Al observar el desarrollo tecnológico y la implementación de técnicas en busca del mejoramiento continuo por parte de quienes lideran procesos y toman decisiones importantes en la empresa, se confirma lo dicho por Heráclito: "No existe nada permanente, excepto el cambio". Las organizaciones, no son la excepción de esta realidad. Por ello, la clave del éxito ayer, no es suficiente hoy. Así, se necesita conocer la organización moderna y que pueda auto-evaluarse en productividad; para descubrir cómo desarrolla procedimientos, que permiten lograr los retos preponderantes de la globalización. En este trabajo, se comparte la realidad operacional de las pequeñas y medianas empresas de casos específicos en Colombia. Estas, en su continua lucha por la sobrevivencia y permanencia en el mercado, incorporan herramientas de calidad para alcanzar su misión y marcar diferencia competitiva, las cuales son un apoyo para las organizaciones, sin embargo, no todas alcanzan la implementación de modelos de calidad, y quienes los aplican generalmente son de procedencia internacional. Este artículo, pone en consideración experiencias vividas en diferentes organizaciones, donde la aplicación de la calidad total no siempre ha arrojado resultados esperados para las organizaciones, lo que permite que se convierta en un caso de estudio de interés. Acá, se muestran varios casos en diferentes organizaciones con la temática, para lo cual se realizó la escritura antológica y anecdótica, desde la experiencia individual y colectiva que ha permitido crecer y comprender que no todas las organizaciones están preparadas para incorporar apropiadamente las propuestas.

Palabras clave: 1. Calidad Total, 2. Productividad, 3. Eficiencia, 4. Ejecución

\section{Abstract}

We live in a changing age. Watching the technological development and the implementation of techniques in search of the continuous improvement by who lead processes and take important decisions in the company, it confirms what Heraclitus said: "There is nothing permanent except change." Organizations are not the exception to this reality. Therefore, the key to success yesterday is not enough today. Thus, it is necessary to know the modern organization and that can self-evaluate in productivity; to discover how it develops procedures, which allow achieving the preponderant challenges of globalization. In this work, the operational reality of small and medium enterprises of specific cases in Colombia is shared. These, in their continuous struggle for survival and permanence in the market, incorporate quality tools to achieve their mission and make a competitive difference, which are a support for organizations, however, not all reach the implementation of quality models, and those who apply them are generally of international origin. This article takes into consideration experiences lived in different organizations, where the application of total quality has not always yielded results expected for organizations, which allows it to become a case study of interest. Here, several cases are shown in different organizations with the theme, for which the anthological and anecdotal writing was done, from the individual and collective experience that has allowed growing and understanding that not all organizations are prepared to properly incorporate the proposals.

Keywords: 1 Total Quality, 2 Productivity, 3 Efficiency, 4 Performance

1 Docente Vinculado Tiempo Completo, Politécnico Colombiano Jaime Isaza Cadavid. Carrera 48 N 7 - 151, oficina P19-150. fjlopez@elpoli.edu.co

2 Docente Ocasional Tiempo Completo, Politécnico Colombiano Jaime Isaza Cadavid. Carrera $48 N^{\circ} 7$ - 151, oficina P19-210. Modulo C. guillermopereira@elpoli.edu.co

3 Docente Ocasional Tiempo Completo, Politécnico Colombiano Jaime Isaza Cadavid. Carrera 48 N 7 - 151, oficina P19-208. Modulo X. jamontoya@elpoli.edu.co

4 Docente Titular, Corporación Universitaria Americana. Calle 50 N43-65, Club Medellín. Bloque 8.dmmontoya@americana.edu.co 


\section{INTRODUCCIÓN}

La gestión de la calidad y la productividad son áreas claves de rendimiento en las organizaciones de producción, cuando no se está preparado o no se hace una interpretación adecuada de las herramientas utilizadas internacionalmente, para logar productividad y calidad en los diferentes procesos organizacionales, se percibe que quienes lideran procesos, llámese directivos o coordinadores de área pueden conducir al deterioro general de los objetivos propuestos, por ende se pone en riesgo el capital de la organización en términos de retorno de inversión. Este artículo pone de manifiesto la experiencia vivida por parte de los autores, en diferentes organizaciones, en áreas como: gestión humana, calidad, productividad y docencia universitaria. Estos compartires, dan lugar al análisis e interpretación de situaciones concretas, ocurridas en la implementación y desarrollo de prácticas apoyadas en las denominadas herramientas de la calidad interpretando el término calidad total, como la integración de esfuerzos de los encargados empresariales, concibiendo la empresa como un sistema integral que genera rentabilidad evitando despilfarro de recursos.

Algunas organizaciones en el contexto colombiano se apoyan y siguen en la búsqueda incesante de modelos de calidad total, los cuales provienen en gran parte de Japón, Estados Unidos y algunos de Alemania, esto con el propósito de lograr la mejora continua y diferencia competitiva en la cadena productiva. Es un cuello de botella la implementación y desarrollo, que tampoco han generado el grado de éxito esperado. Las discusiones suscitadas de las experiencias, muestran la compleja realidad aplicativa de las variables de productividad y calidad, los efectos colaterales que ocasiona la implementación de modelos internacionales en empresas latinoamericanas muestran en los estudios realizados evidencias de costos y pérdidas. Teniendo en cuenta que la mayoría de las organizaciones en Colombia, son medianas y pequeñas empresas que se reconocen como (PYMES), éstas poseen recursos limitados,

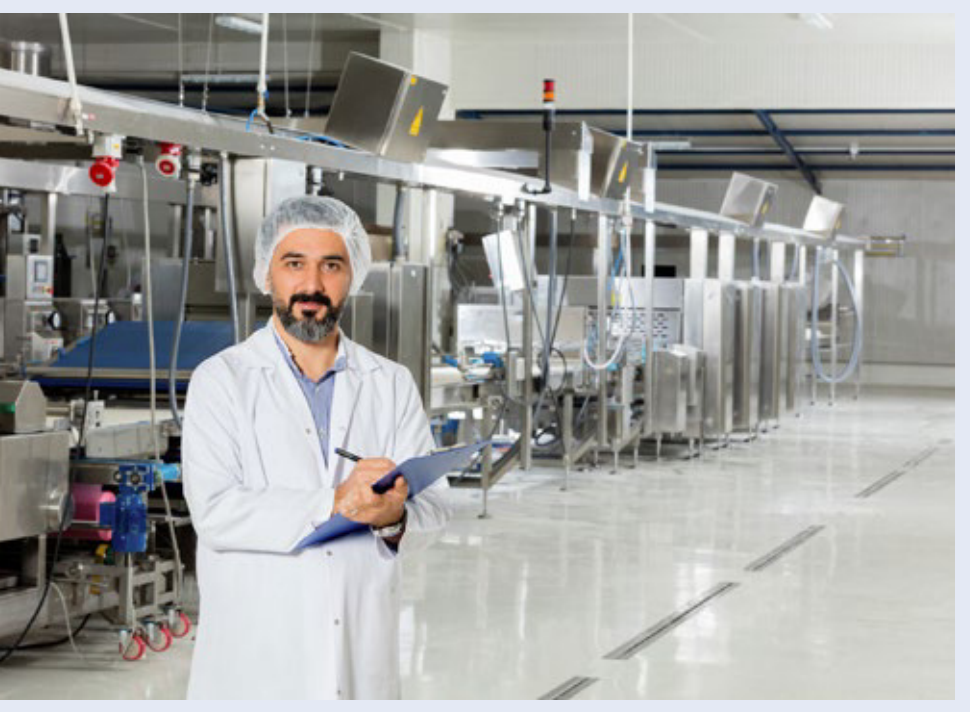

lo cual denota tecnología incipiente, que no ayuda a la optimización de los recursos y por ende las pone en desventaja competitiva respecto al mundo.

\section{METODOLOGÍA}

Se hizo una recopilación de experiencias, de un equipo interdisciplinario conformado por docentes universitarios, y profesionales con una amplia práctica en los temas tratados. Se documentaron los casos vividos además de entrevistas realizadas a expertos en alta dirección y control de producción, se realizó de igual forma un estudio bibliométrico sobre el estado situacional en la productividad y calidad total.

Es una investigación que parte del análisis cualitativo, relacionado con una muestra de empresas de producción y servicios, la principal característica que da lugar al estudio, son los comportamientos actitudinales de quienes han intervenido de una u otra forma en los procesos de mejoramiento continuo, y que en el estudio de indagación resaltan las herramientas internacionales de calidad total, sin medir a profundidad el impacto que traen consigo las decisiones apoyadas en la intervención de procesos estandarizados por entes reguladores en la calidad. Es una conclusión de los autores: las herramientas, los algoritmos y los flujos de proceso no podrán sustituir el pensamiento estratégico, ya que, si sólo se apela a lo primero, la gestión puede ser formal y fría, convirtiéndose en tecnócrata y por tanto no creíble. Finalmente se describen algunos casos dentro de las organizaciones muestra cómo la visualización del obstáculo principal para modificar la forma de gestionar es desde la experiencia, los escasos recursos, la ausencia de liderazgo y por supuesto el desarrollo de la cultura organizacional.

\section{RESULTADOS Y DISCUSIÓN}

Como se indicó anteriormente, se deduce que las organizaciones hoy en día, tienen dificultades en cuanto productividad y calidad en casos específicos no se tiene confiabilidad, se da poca utilización, y se refleja desconocimiento de las herramientas básicas de mejoramiento continuo, basados en Gutiérrez $(\mathrm{H}$. Gutierrez, 2008), donde cita a Demming, quien expone: "...con planes que ataquen las causas fundamentales, que desarrollen proyectos para abordar los problemas más importantes mediante el ciclo PHVA", que apoyan de manera permanente, la mejora de la calidad, además es allí donde (H. Gutierrez, 2008), “... se estarán dando los pasos necesarios, para asegurar el punto más importante del éxito de un programa de mejora de la calidad: la constancia en el propósito de mejorar el producto", la cual es un ítem no negociable, en ningún proceso, pues la calidad es dar satisfacción a los requerimientos de los clientes, tanto internos como externos.

Lo anterior permite generar una discusión comparativa sobre los antecedentes y estado situacional de la productividad y la calidad. En la revolución industrial, el 


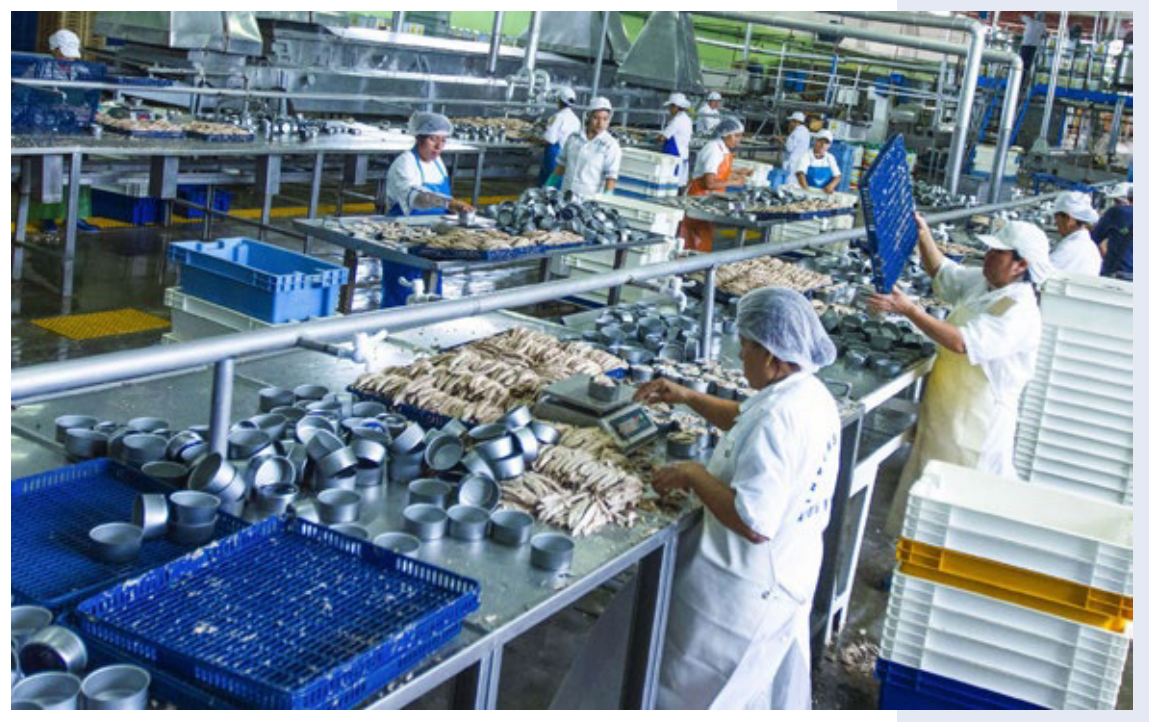

el cumplimiento de sus especificaciones o requerimientos. Los colaboradores, son una fuente inagotable de ideas de mejoramiento de procesos, sólo si en las organizaciones se presentará un nuevo estilo de liderazgo, "...de dirección comprometido profundamente con la filosofía de mejora continua de procesos, acorde con la necesidad de trabajo en equipo y con la innovación y que además articule una amplia comunicación sobre la calidad y la mejora en la organización" (H. Gutierrez P.,2008).

De otro modo, aunque existe diferencia entre el concepto de calidad y productividad desde las diferentes ópticas teóricas por parte de los expertos de la calidad, en la vida práctica se haya que ambos tópicos están estrechamente

punto de vista productivo representó la transformación del trabajo manual por el trabajo que bien se podría llamar mecanizado. Anterior a esta época, el trabajo se hacía de manera artesanal, y es así, entonces como la calidad va pasando por diferentes etapas, la calidad se inicia con la idea de hacer hincapié en la inspección, tratando de no sacar a la venta productos defectuosos. Poco tiempo después, se dan cuenta de que el problema de los productos defectuosos radicaba, en las diferentes fases del proceso y que no bastaba con la inspección estricta para eliminarlos. Por esta razón es que se pasa de la inspección al control de todos los factores del proceso, abarcando desde la identificación inicial hasta la satisfacción final de todos los requisitos y las expectativas del cliente.

En el caso específico del análisis, la productividad y la calidad dentro de las (Pymes) pequeñas y medianas empresas. La mejora continua, es un asunto fundamental para mantenerse competitivo en el mundo globalizado. Del mismo modo, cuando se incorporan estos modelos indiscriminadamente en dichas organizaciones, el grado de asertividad no es positivo. Es un error recurrente implementar una herramienta con el sólo hecho de que haya funcionado en otro contexto organizacional, cada una de ellas tiene su propia cultura.

Es otro foco encontrado en los análisis realizados de este estudio, aquellas organizaciones que no utilizan las herramientas de mejoramiento, tienden a desconocer las bondades de las mismas, justifican la falta de productividad y algunas veces de calidad, señalando a los colaboradores como responsables de dichos resultados, por tanto, las consecuencias son despedir a los trabajadores que aparentemente están generando los bajos resultados organizacionales, decisiones tomadas sin fundamento; es de recordar que los colaboradores son los ejes fundamentales en las empresas, pues son ellos quienes tienen el conocimiento y la habilidad necesaria, para la ejecución de las actividades que nos acercan a entregarle al cliente (interno o externo), el producto bajo relacionados cuando de mejoramiento de procesos se trata. Trayendo a colación la exposición de Cruelles (J. Cruelles, 2018), donde dice como"Una PYME debe disponer de un sistema de cálculo de costes industriales científico y actualizables en función de parámetros con el cual se debe conocer de manera certera los costes de los productos". De la teoría anterior se comparte algunas alertas de Pymes de Latinoamérica que carecen de los datos reales de sus costos industriales con los cuales determinar un precio de venta adecuado, esto porque las técnicas empleadas aportan datos parciales sobre la producción y la inmediatez, la toma de decisiones organizacionales con pocos datos, hace que muchas de ellas finalmente estén tomando una decisión drástica, cerrar puertas, pues no son productivas y no estan cumpliendo con la meta organizacional de ganar dinero.

Sin embargo, las teorías aplicadas de una manera racional, sin olvidar la disciplina que debe primar en cualquier cambio que se desee implementar y máxime en lo que a productividad se refiere. Experiencias vividas han demostrado las bondades que se pueden generar en términos de competitividad, no sólo con la aplicación de fórmulas exitosas en otras latitudes sino, logrando la participación activa de todos los dueños de los procesos, es decir, las personas motivadas y tenidas en cuenta en las transformaciones funcionales empresariales (OIT, 2006) "la planificación de la producción se facilitará si una buena política de personal y un sistema de incentivos bien aplicado alientan a los trabajadores a ser cumplidores", señala esto que mientras más motivado el equipo de trabajo, mejores serán los resultados, ejemplo: en una de las compañías donde se han prestado los servicios profesionales, se logran indicadores altos de productividad formando equipos de trabajadores con conocimientos interdisciplinarios, para generar ideas que dieran soluciones posibles y simples a problemas de proceso o ideas innovadoras que mejoraran la eficiencia en la planta de producción. Se expresa que: 
"La vinculación al grupo de trabajo es emocional. El empleado forma parte de la empresa, que es un ente unido y único. El empleo de por vida ha sido la praxis del sistema de gestión japonés en las grandes empresas" ( $M$. Rajadell , 2010). Lo destacable en esta forma de elevar la productividad radica básicamente en lo útil que se sienten los empleados cuando se les tiene en cuenta para generar los cambios, además, son más viables las transformaciones cuando se generan autónomamente. De otro lado, se incentiva al personal de forma cualitativa con participación y reconocimiento como individuo aportante al logro de objetivos organizacionales con presencia de familiares más cercanos, en eventos donde se exaltan los mejoramientos.

Si nos centramos en la autonomía de los trabajadores, y tomamos como ejemplo una planta de producción, en el alistamiento de los equipos, las reparaciones menores, los cambios de referencia, llevan a la empresa a disminuir tiempos perdidos en el proceso, sin tener que hacer ningún tipo de inversión, ni pagar asesores externos y expertos, son los mismos trabajadores que han estado allí por largos periodos de tiempo, ya que quien mejor conocimiento posee del puesto de trabajo es el mismo operario. Lo que nos lleva a compartir el pensamiento de Palacios (L.C, 2009) "El conocimiento de las capacidades humanas ayuda a mejorar las observaciones, evaluarlas, desearlas, diseñarlas y producirlas; tal conocimiento, caracteriza a las personas como benefactores y componentes de los sistemas que ellas mismas diseñan, construyen y emplean". Así que el problema no es generar grandes erogaciones de dinero para inversiones en bienes de capital, sino en darle importancia al capital humano que poseen las PYMES, pues este capital posee, conocimiento.

Otro aspecto interesante consiste en empoderar los trabajadores en el desempeño de sus labores cotidianas con liderazgo, en la toma de decisiones respecto de la calidad y productividad sin estar dependiendo de las jefaturas de coordinadores de línea, fomentando el trabajo en equipo, como lo plantea Deming en sus catorce principios gerenciales sobre calidad, cuando se señala que en el Premio Nacional de Calidad de México (PNCM), se afirma: "Los líderes, a través de su ejemplo, deben servir de modelo con su comportamiento ético y congruente, al involucrar, comunicar, dar asistencia y apoyo, así como al desarrollar a futuros líderes, revisar el desempeño de la organización y reconocer el desempeño de los empleados" (H. Gutierrez P.,2008), esto es darle al colaborador la importancia que tiene en su labor. De lo anterior se deduce la hipótesis de considerar la empresa como un sistema integral productivo, para el logro de objetivos estratégicos, con la participación de cada uno de sus empleados sin hacer diferencia del rango o función desempeñado, considerando la sinergia del talento humano de una manera holística, aprovechando las habilidades y el entrenamiento constante y de esta manera propiciar la mejora continua en las organizaciones.

En este orden de ideas en lo referente a calidad Juran

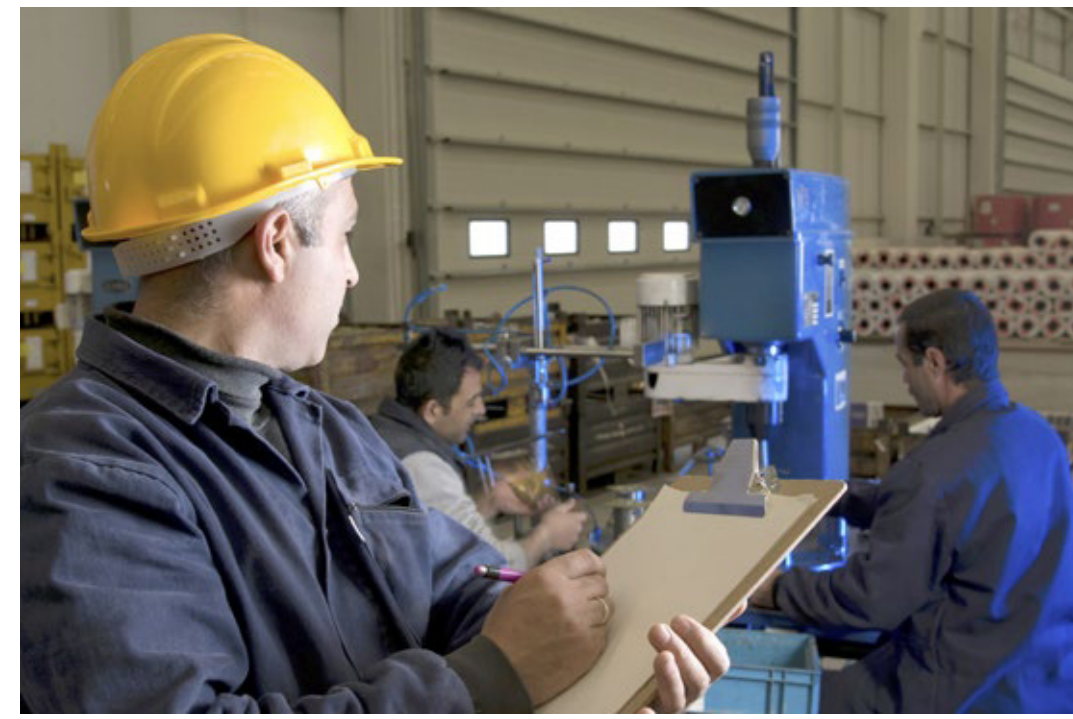

enfatizo, la responsabilidad de la administración para mejorar el cumplimiento de las necesidades de los clientes, con un enfoque muy particular sobre la administración de la calidad, que llamo trilogía de la calidad, que divide el proceso de la administración de la calidad en tres etapas; planeación ,control y mejoramiento de la calidad, “...el control de la calidad busca caracterizar qué tan lejos se está del nivel planeado y analizar los cambios en los procesos." (H. Gutierrez P, 2014).

Por su parte, la American Society for Qality (ASQ) señala: "calidad es un término subjetivo para el que cada persona o sector tiene su propia definición" (ASQ , 2018). De otro lado la norma ISO -9001:2015, define calidad como "...el grado en el que un conjunto de características inherentes cumple con los requisitos", (J. A. Gómez, 2009), entendiendo requisito como una necesidad o expectativa establecida, generalmente implícita u obligatoria. Para el equipo de trabajo se considera que la calidad empieza y termina con el cliente, como bien afirma Deming, referenciado por (H. Gutierrez P., 2014), "...la calidad la proporciona primeramente el sistema, y de esto es responsable la alta dirección".

Volviendo a la productividad propiamente dicha, refleja los resultados que se obtienen en un proceso, la medición de la productividad resulta de valorar adecuadamente los recursos empleados para producir o generar ciertos resultados. En la discusión frente a las vivencias en las empresas relacionadas con los componentes de calidad y productividad hay coincidencias que bien se puede ser eficiente y no eficaz o viceversa, la efectividad es el punto central del objetivo.

Avanzando en el tiempo se encuentran organizaciones productivas, que buscan el crecimiento y permanencia en los mercados, logrando el apoyo voluntario de sus empleados, no sólo entregándoles la autonomía en la toma de decisiones, sino que, al mismo tiempo le asignan participación de la rentabilidad obtenida con el trabajo mancomunado. Aquí, vale la pena hacer una pequeña 
digresión, sobre la timidez empresarial a la hora de compartir las utilidades con los colaboradores, ya que, aún se manejan paradigmas egoístas sobre riesgo de capital versus la productividad obtenida, pues se siguen describiendo situaciones conservadoras consideradas obsoletas, además, no hay constancia en la aplicación de técnicas de mejora continua; en la mayoría de los casos se hace de una forma puntual o por decirlo de otro modo, obedece más a una moda que a una política. Es así que, cuando se hace memoria sobre el impacto que tiene el incorporar modelos de calidad en el contexto empresarial el esfuerzo se centra en la mejora continua; el devenir cotidiano para las empresas es: productividad, eficiencia, eficacia en la ejecución operacional en todas las áreas del sistema organizacional acompañado de los procesos de la calidad. Es así, que al dar una mirada a los resultados obtenidos de los casos tratados en este estudio, se permite inferir sobre la realidad operacional enfrentada, desde una perspectiva general se obtuvieron resultados positivos en la implementación, otras se quedaron en el intento; estas últimas, presentaron pérdidas significativas; todo parece indicar que por el afán de sobrevivir y ser competitivo, no tomaron en cuenta las expectativas de innovar en sus procesos respaldados por herramientas de calidad.

Es así como Gastón señala en su discurso referenciando a (J. Masini, 2018) que: "El que opta por Hoshin Kanri, es porque lo ha comprendido, cree fielmente en él y tiene la firme voluntad de implantarlo". Bajo este contexto, sucede que se debe tener la información necesaria para su implementación y las facilidades dentro del entorno en el que se desenvuelve una organización, para lograr el éxito previsto, así como la creación de disciplina y cultura organizacional, trabajo en equipo. Del comportamiento observado en el campo organizacional, se ha identificado un índice alto de directivos que, buscando reconocimiento justifican entrar en la moda de la calidad total, sin criterio sugieren y afirman que los modelos de calidad total están diseñados sólo para compañías con niveles de inversión y de producción suntuosos, pero la experiencia les muestra que es también cuestión de compromiso de todos los miembros de la organización.

Es así como se puede apreciar hoy en día que las organizaciones sin importar el tamaño, enfrentan grandes dificultades en cuanto Productividad y Calidad se refieren, consideraciones que en algunas se da por la poca o inadecuada utilización de las herramientas y se observa que, gran parte de la responsabilidad recae en quienes la gestionan. Teniendo en cuenta otros aspectos, también han existido quienes se oponen y se resisten al cambio y cuestionan la efectividad de los resultados esperados. No obstante, para enfrentar la incertidumbre se hace necesario conocer los retos de la organización moderna y poder así adentrarse en una auto-evaluación, que permita descubrir el cómo cumplir con los retos organizacionales. Se concuerda que la mejora continua, es un asunto fundamental para mantenerse competitivo en el mundo de los negocios.

En este apartado los autores expresan que la organización al incorporar modelos indiscriminadamente en la calidad, el grado asertividad no siempre es positivo en términos de resultados esperados. Es un error recurrente e irresponsable, introducir herramientas de mejora, sin

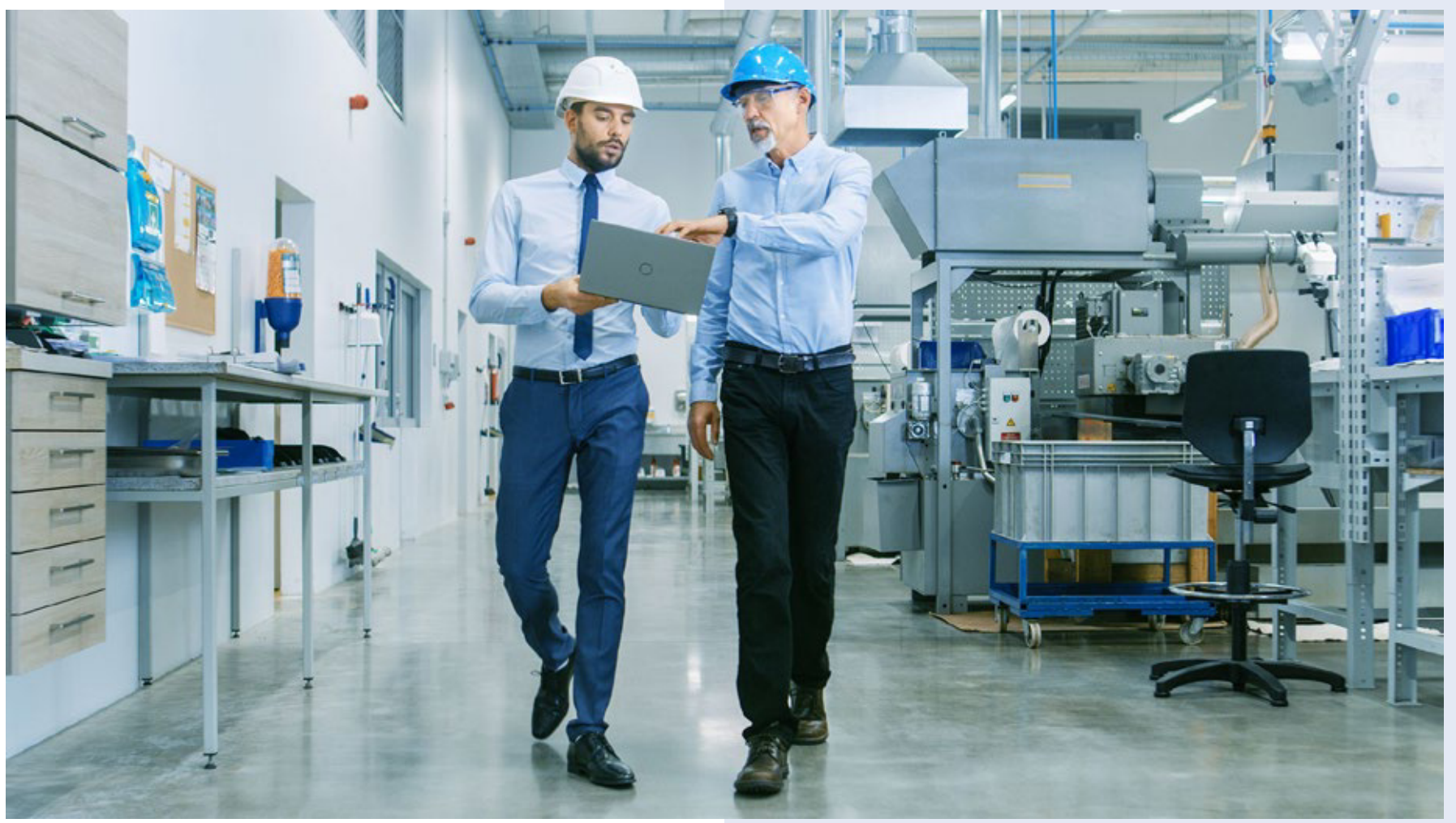


los estudios previos que permitan determinar, si se está en capacidad de asumir el reto, se entiende que no basta sólo con pensar estratégicamente. La práctica deja ver claramente la diferencia entre pensamiento estratégico y planeación estratégica. Según (Área de Comercialización, Investigación de Mercados, 2017) referenciando a Kotler "...la planificación consiste en decidir hoy lo que va a hacerse en el futuro", es decir, comprende la determinación de un futuro deseado y las etapas necesarias para realizarlo. Para lo cual se debe modelar y repensar las áreas de negocio, garantizando la generación de valor agregado. De la teoría anterior, los autores intervienen dando la importancia a adelantar procesos de vigilancia estratégica, encaminados a reconocer los cambios del entorno, que obligan a implementar estrategias a fin de posicionarse en el mercado. En este sentido, las organizaciones deben fijar la misión y establecer las unidades estratégicas de negocio. Lo anterior implica un proceso sistémico que garantice la continua evaluación y monitoreo, dando paso a un círculo virtuoso que garantice anticiparse a los competidores mediante una gestión responsable. No se puede dejar de lado el modelo propuesto por (Steiner, 2017), en tanto que propone en su estructura el desarrollo de una planificación transversal que abarca decisiones a nivel directivo, táctico y operativo; los cuales garantizan un despliegue de los objetivos a todo nivel, articulando esfuerzos para que los eslabones estén cohesionados y sus acciones sean consistentes con la misión organizacional de manera holística. Sin

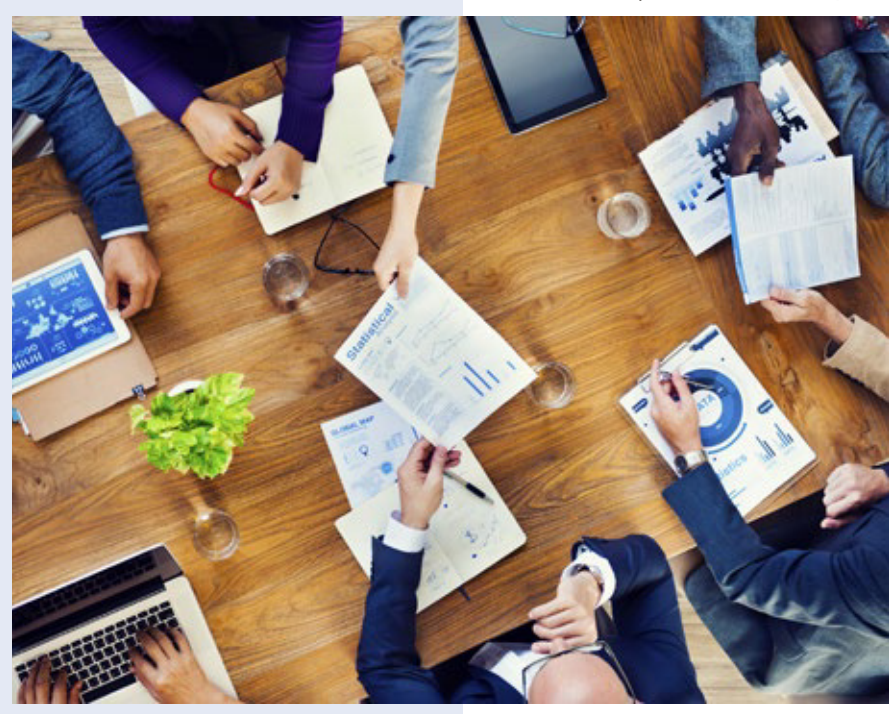

organizaciones donde se ha vuelto incesante la búsqueda de productividad tendiente a la calidad total. Estos casos reflejan un panorama de la realidad operacional, que se vive en un momento dado en empresas de producción y de servicios. Los nombres de las compañías no aparecen mencionados, en aras de la discreción y el respeto por dichas organizaciones, sólo se describe el sector industrial al cual pertenecen y los casos que causaron mayor discusión para los autores.

\section{Caso 1:}

En una empresa metalmecánica, un operador al hacer su labor detectó una oportunidad de mejora y la expresó a su líder de proceso, el cual a su vez escalo el tema; la situación detectada es la siguiente: un producto cuya materia prima tenía un costo de U\$2.= por kilo, tiene un proceso que contempla varias operaciones antes de llegar a ser producto terminado; la materia prima consistía en una lámina cuyas medidas eran $330 \mathrm{~mm}$ de largo por $290 \mathrm{~mm}$ de ancho y $2 \mathrm{~mm}$ de espesor, con un peso de 590 gramos, es decir, 0,590 kilos. La primera operación es aplicar sustratos o recubrimientos por ambas caras de la lámina, luego se transportan las piezas al proceso siguiente, aproximadamente 30 metros, (lote normal de trabajo, 1000 unidades, Cantidad óptima), el siguiente ciclo de trabajo es troquelar un desarrollo, el cual genera un $17 \%$ de desperdicio, tanto de material como de recubrimientos, esto adicionalmente genera una operación más que es pulir los bordes, pues el mantenimiento del troquel no es tan ajustado y al momento de troquelarse el desarrollo para el producto, presenta rebabas, (bordes disparejos); para continuar el proceso de fabricación se debe pulir y en este momento se encuentran más desperdicios, pues al ser una actividad netamente manual, al contacto del desarrollo con el papel de esmeril, se dañan o hacen marcas en el recubrimiento, por tanto incremento en el desperdicio.

La acción de mejora presentada fue hacer la solicitud al proveedor de entregar el desarrollo, no la lámina, como en el momento se recibía. Esto desencadenó una serie de consultas tanto internas como en el proveedor, hasta que al fin se obtuvo una respuesta preliminar para tomar decisiones en pos del mejoramiento, el provisor puede hacer caso a la solicitud, siempre y cuando el fabricante cancele un valor de unos millones de pesos para obtener los troqueles que permitan al abastecedor entregar según las necesidades del productor. En la organización se evalúa 


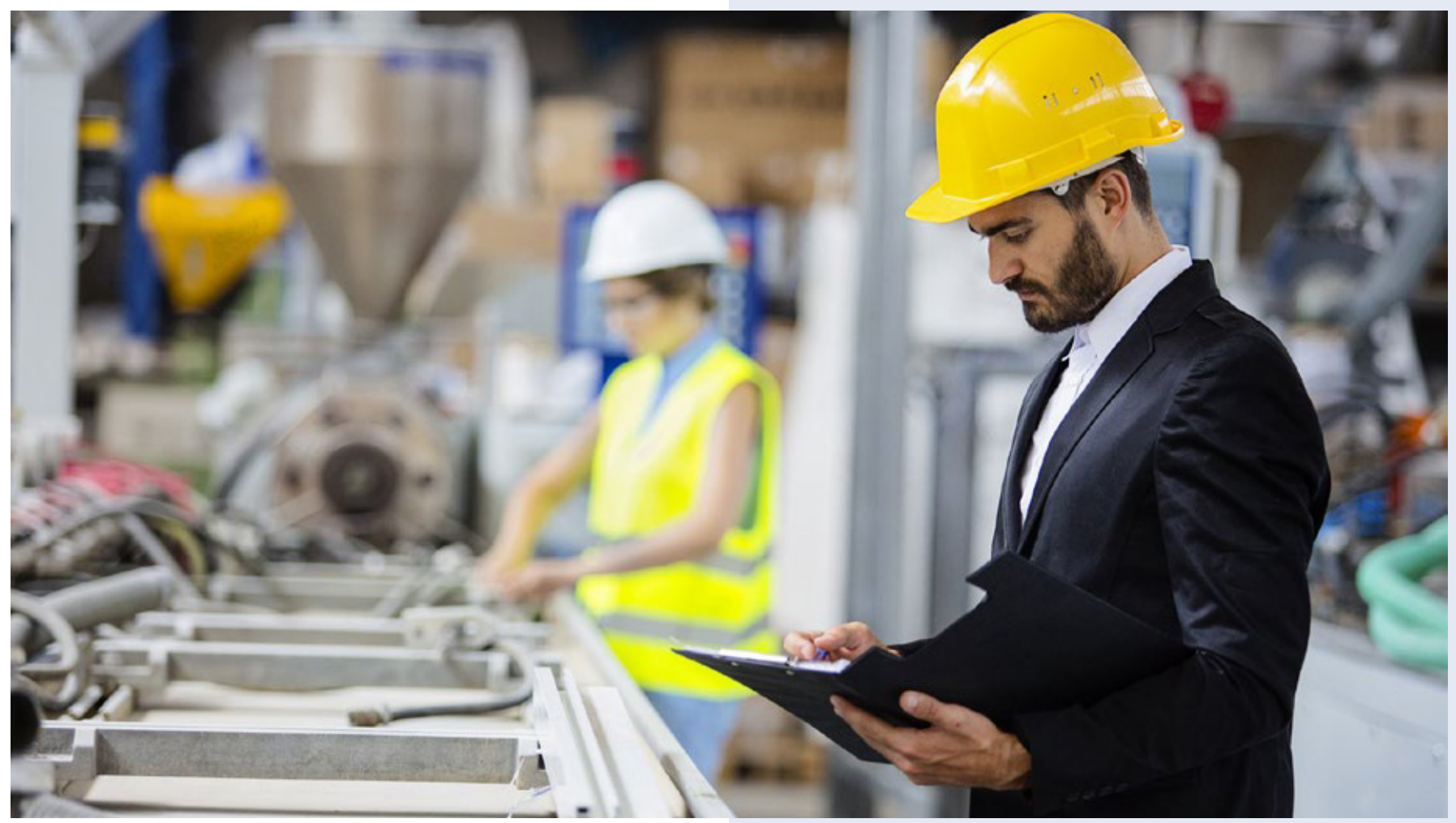

y se determina que, con el porcentaje de desperdicio de material, la Tasa Interna de Retorno de la inversión (TIR), será de doce meses, al nivel de producción y ventas actual, con esto se toma la decisión de hacer el trámite, cancelar los dineros al proveedor para que proceda a construir los troqueles y seguir enviando al productor el desarrollo solicitado. Las mejoras alcanzadas son: disminución del $17 \%$ de desperdicio, equivalente a 4800 kilos por año, la calidad del producto, lo cual no se había contemplado, mejoró, por tanto, la satisfacción de los clientes finales aumentó y así se incrementaron las ventas en un 50\%, también se evitaron las operaciones de Troquelar y pulir el desarrollo, así la TIR real fue de 5,6 meses.

\section{Caso 2.}

En una organización, el directivo de planeación, justificaba la baja productividad y calidad de su gestión, culpando a los colaboradores de su área, señalándolos como responsables únicos de no alcanzar los objetivos; en las reuniones de gerencia donde se discuten los logros, sugería despedir a los trabajadores que tenían bajo desempeño. La sugerencia de toma de decisión del directivo no contempla factores que afectan el liderazgo; donde no dejan de presentarse ciertos síndromes que pueden menoscabar las intenciones productivas de la organización. Aquí se resalta lo descrito por (J. Aguilera, 2002) al referenciar a Eric Flamholtz, acerca del Síndrome del pigmeo, "La característica típica que señala a este tipo de líder es el profundo temor que le provoca los subalternos más creativos o más preparados que él, considera que: aquella persona inquieta en el equipo, ¿Es una amenaza a su autoridad? por ello, busca minar su afán creativo o simplemente eliminarlos del grupo". Este tipo de síndrome genera equipos de bajo rendimiento y autoestima, fácilmente controlables, con rendimientos mediocres y pobres procesos de innovación. Por fortuna, existe un buen número de directivos no diagnosticados con síndromes de liderazgo; es de recordar que los colaboradores son los ejes fundamentales en las empresas, pues son ellos quienes tienen el conocimiento y la habilidad necesarias para la ejecución de las actividades que acercan a entregarle al cliente (consumidor final), el producto bajo el cumplimiento de sus especificaciones o requerimientos. Los colaboradores, son una fuente inagotable de ideas de mejoramiento de procesos y es por eso que se deben desarrollar, para que puedan crecer en la organización y como consecuencia, esta tenga rentabilidad y permanencia en el mercado, dando lugar a la generación de empleo formal, con perspectivas de crecimiento económico y social, tanto para los colaboradores como para la misma organización.

En el caso anterior, la alta gerencia sólo sugirió un cambio de actitud frente a sus colaboradores a manera de prueba piloto, logrando en 3 meses un incremento de la productividad del $20 \%$.

\section{Caso 3.}

La autonomía de los trabajadores en una empresa de producción de pastas para frenos de vehículos se reflejó un asunto de éxito, esto se vivió en la planta de producción con 
el alistamiento de los equipos, las reparaciones menores y los cambios de referencia, llevando a la empresa a disminuir tiempos perdidos en el proceso de más o menos un 15\%; esto se da gracias a que se empoderó a los operadores de la máquina y es así como se logran ahorros significativos en producción sin tener que hacer ningún tipo de inversión ni pagar asesores externos, una vez más, se resalta que quien mejor conocimiento del puesto de trabajo posee es el mismo operador. De modo que, el problema, no es generar grandes erogaciones de dinero para inversiones en bienes de capital, sino en darle importancia al talento humano; esto es resultado de escuchar más a los empleados, y multiplicar el conocimiento.

\section{Caso 4.}

Un caso similar al anterior, es el de una empresa transportadora, la cual con el aporte de conocimiento de los trabajadores disminuye las distancias recorridas en el proceso de recogida y entrega de mercancías, sólo con rediseño de ruta y tipo de vehículo utilizado se incrementa en un $8 \%$ la productividad. Esta mejora, fue propuesta por un empleado encargado de la logística, quien no tenía un cargo considerado gerencial en la organización. Empresa donde algunos empleados humildemente expresan que los directivos toman decisiones sin tener conocimiento de su hacer.

\section{Caso 5.}

Otra experiencia dentro de la antología de vivencias es donde uno de los autores presencia en una industria de Metalmecánica el ahorro en materiales gracias al apoyo de los operarios en la disminución del desperdicio del Latón (aleación de cobre y zinc), equivalente al 12\%; simplemente con la modificación del paso de la lámina por la troqueladora (mermar el halado de la lámina en el proceso de troquelado), como se le conoce en este tipo de industria, esto condujo a una mejora substancial en el proceso y ahorros significativos para la organización.

\section{Caso 6.}

Este es el caso de una industria de alimentos, que tiene relación con más de 25 países, la cual se dedica al procesamiento, ensamble y venta de comidas rápidas. En su programa de calidad, contrató una compañía externa de servicios, para qué mediera la calidad del servicio al cliente, ofrecido en las diferentes tiendas de EE.UU. Esta compañía contratada, utiliza un instrumento de medición conocido como Momentos de Verdad. Dicho instrumento, tiene como indicadores de medición del servicio: tiempo para recibir la orden de compra, cortesía por parte de quien toma la orden. El cuestionario, contiene preguntas tales como: ¿Fue bienvenido con un comentario amable?, ¿Le agradecieron su compra de forma amable, ofreciendo un comentario sincero de despedida y haciendo uso visual directo?, entre otras. Este instrumento, es administrado por una persona de la empresa subcontratada, denominada -cliente anónimo-; teniendo en cuenta la puntuación alcanzada por el vendedor, la prestadora del servicio, genera unas recomendaciones, las cuales se tienen en consideración para los planes de mejora. El asunto es, que el $70 \%$ de las tiendas puntuaban bajo, lo cual sugería una investigación exhaustiva que permitiera determinar las causas de dichos resultados recurrentes. Al efectuar entrevistas al personal, reuniones periódicas con los líderes de grupos, se halló como resultado que el $85 \%$ del personal estaba desmotivado percibían la organización como un sitio no acogedor, laboraban allí mientras solucionaban dificultades económicas es decir como un lugar transitorio; pensaban que a la empresa no le importaban los empleados y se sentían mal remunerados.

Luego del análisis del instrumento utilizado, se desarrollaron planes motivacionales y se les dio mayor participación en la toma de decisiones, posteriormente se evaluó de nuevo, logrando mejoras en dicha percepción hasta en un $50 \%$, comparándola con los resultados anteriores.

En la siguiente tabla con su respectivo gráfico se hace un resumen del porcentaje de mejora obtenido por cada caso, y su calificación de acuerdo al puntaje.

\section{Tabla 1. Inclusión del Talento Humano en las Decisiones Organizacionales.}

\begin{tabular}{|c|c|c|}
\hline $\begin{array}{c}\text { CASO } \\
\text { DE ANALISIS }\end{array}$ & $\begin{array}{c}\% \text { DE } \\
\text { MEJORA }\end{array}$ & VARIABLE \\
\hline 1 & 17 & Materia prima \\
\hline 2 & 20 & Productividad \\
\hline 3 & 15 & Tiempo \\
\hline 4 & 8 & Productividad \\
\hline 5 & 12 & Materia prima \\
\hline 6 & 50 & Motivación \\
\hline
\end{tabular}

FUENTE: Elaboración propia.

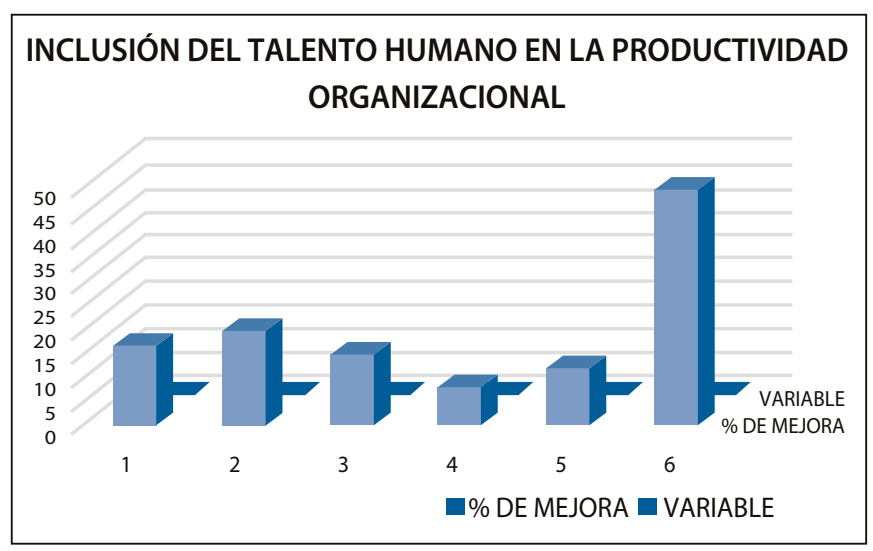

Figura 1. Inclusión del Talento Humano en La Productividad Organizacional.

FUENTE: Elaboración propia. 
Se observa, como la participación del talento humano en las decisiones estratégicas y tácticas empresariales, redunda en el beneficio común a nivel de productividad y calidad, además que mejora el clima organizacional en todos los niveles jerárquicos de las compañías.

Se puede inferir con los casos mostrados donde se evidencia a través de la praxis resultados tangibles en el incremento en ventas, aprovechamiento de recursos como el tiempo, el espacio, la materia prima que brinda resultados tangibles en productividad. Cabe destacar también, como el talento humano responde positiva o negativamente ante los estímulos brindados por las PYMES a través del empoderamiento y la participación activa en la toma de decisiones.

\section{CONCLUSIONES}

La calidad se logra en la medida en que la organización sabe estudiar los procesos y mantenerlos bajo control. Hacer esta tarea requiere que se dé participación a las personas que están más cerca de los procesos. Para ello habrá que capacitarlos y darles instrumentos que les sirvan para identificar los problemas.

Es una organización humana, donde la comunicación consulta la participación y los sentimientos de las personas y son tomadas en consideración, esto forma parte del empoderamiento de la gente en el trabajo.

Se enfatiza en el manejo efectivo de la información, donde las decisiones se toman a base de datos y, además, se le da importancia a la búsqueda de información, se espera que las opiniones estén documentadas; este es el nuevo lenguaje de las organizaciones que aprenden

Las organizaciones que no interpretan la necesidad de proclamar sus objetivos, fijar sus esfuerzos para establecer la ruta ideal para alcanzarlos, posiblemente tardarán en lograrlos, a diferencia de aquellas que hagan una lectura seria de sus intereses y de las variables exógenas y endógenas, para así definir y redefinir- si es el caso- los planes de acción que garanticen el cumplimiento eficaz de las metas.

Se cree que el liderazgo, más que una tendencia mundial para lograr el éxito de una organización, es un reto preponderante que tienen las empresas del futuro, las industrias proactivas se plantean el cambio cuando evalúan a diario sus competencias. Pues sólo, si en las organizaciones se presentará un nuevo estilo de liderazgo, comprometido profundamente con la filosofía de mejora continua de procesos, que sean acordes con el trabajo en equipo, con la innovación y que articule una amplia comunicación sobre el compromiso colectivo, se considera que es una manera apropiada de hacer diferencia competitiva.

En el contexto organizacional todavía hay mucho que aprender en la construcción, el análisis, la interpretación y la aplicación de las herramientas para la búsqueda de la calidad total.

\section{BIBILIOGRAFÍA}

[1] J. Cruelles,(2017). «Cálculo de Costes Industriales,» 1312 2017. [En línea]. Available: www.zadecon.es. [Último acceso: 1602 2018].

[2] M. Rajadell y J. Sánchez, Lean Manufacturing, (2010). La evidencia de una necesidad., Ediciones Diaz de Santos., , pp. 7-8.

[3] American Society for Quality (ASQ).(2018). «The global vice of quality,». [En línea]. Available: https://asq.org/qualityresources. [Último acceso: 12 Abril 2018].

[4] J. Masini, (2010). «Mundanai Online,» Instituto Mundanai, 5 Mayo 2010. [En línea]. Available: http://www.mundanai. org/2010/05/hoshin-kanri-a-que-no-sabes-lo-que-es-peroseguro-sabes-lo-que-es-el-balanced-scorecard. [Último acceso: 5 Mayo 2018].

[5] H. Gutierrez P. (2008)., Calidad Total y Productividad, Segunda ed., México: McGraw-Hill Interamericana, , p. 46.

[6] H. Gutierrez, (2008). Calidad Total y Productividad., Segunda ed., México: McGraw-Hill Interamericana, , p. 38.

[7] Organización Internacional del Trabajo (OIT). (2006)., Introducción al Estudio del Trabajo, 4a. Edición revisada ed., México: Limusa, S.A. de C.V., 2006, p. 13.

[8] C. Palacios, (2009). Ingeniería de Métodos, movimientos y tiempos., Primera ed., Bogotá: Ecoe, Ediciones, p. 160.

[9] J. A. Gómez. (2017). Guia para la aplicacion de ISO 9001:2015, Bogota: Anor Ediciones.

[10] I. Nonaka. (2017). «La empresa creadora de conocimiento,» Japon,.

[11] H. Gutierrez P. (2017)., Calidad y productividad, Cuarta ed., México D. F.: McGraw- Hill - Interamericana Editores, S.A DEC.V, , p. 32.

[12] Área de Comercialización, Investigación de Mercados. (2018)., «Planeación Estratégica de Marketing.,» [En línea]. Available: http://www4.ujaen.es/ emurgado/tema3.pdf. [Último acceso: 27 Abril 2018].

[13] J. Aguilera. (2012). «Elempleo.com,» 28 Junio 2002. [En línea]. Available: http://www.elempleo.com/co/noticias/ mundo-empresarial/sindromes-de-comunicacion-queafectan-su-liderazgo-1866. [último acceso: 01 Marzo 2018].

[14] Steiner (2017)., Planeacion estrategica loque todo director debe saber, primera ed., Madrid, El galeon: Patria, pp. 62-63.

[15] H. Gutierrez P. (2017). de Calidad y Productividad, Cuarta ed., Mexico, McGrawHill Education, , p. 44.

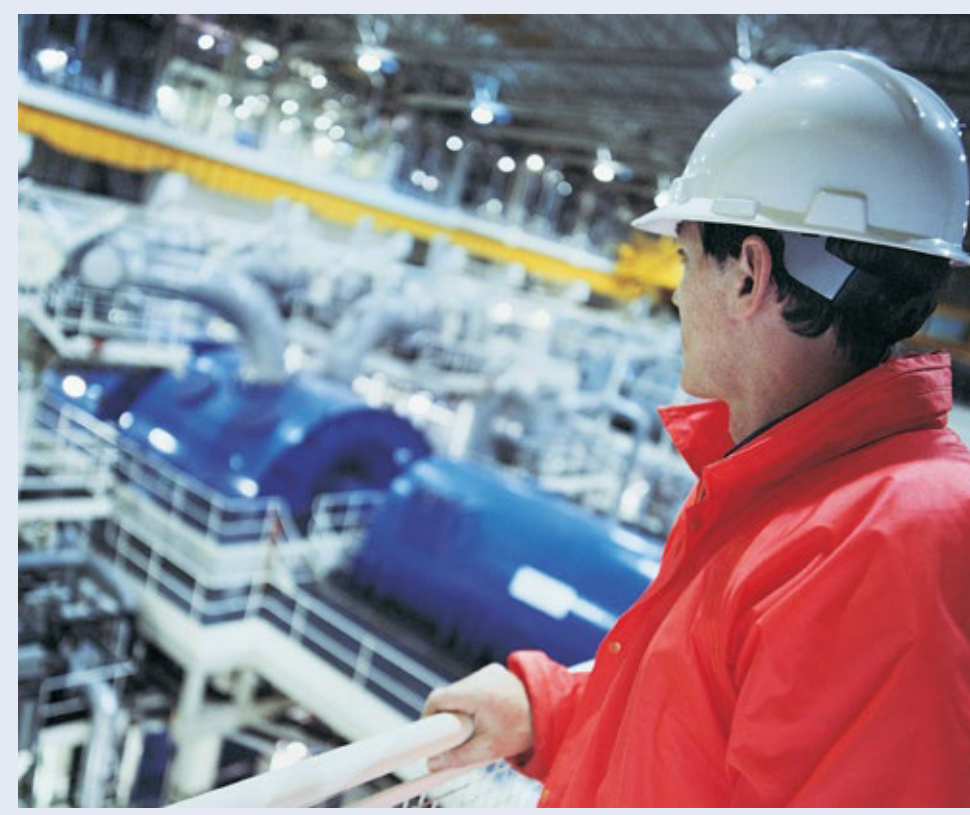

